\title{
Efeito da Linhagem Citoplasmática sobre a Produção de Leite em Bovinos da Raça Caracu ${ }^{1}$
}

\author{
Luciele Cristina Pelicioni' $2,3,4$, Sandra Aidar de Queiroz 2,5
}

\begin{abstract}
RESUMO - O objetivo do presente estudo foi avaliar o efeito da linhagem citoplasmática sobre a característica produção de leite de 11.163 lactações de vacas de um rebanho da raça Caracu. A linhagem citoplasmática foi estudada traçando-se o pedigree dos animais até as fêmeas fundadoras do rebanho. Com o intuito de apresentar estimativas de (co)variâncias e parâmetros genéticos para a característica produção de leite, incluindo-se ou não os componentes de efeito materno e de linhagem citoplasmática, foram realizadas análises univariadas sob seis diferentes modelos. As análises estatísticas foram elaboradas pelo método de máxima verossimilhança restrita livre de derivada. A estimativa da variância de linhagem citoplasmática nos dois modelos que incluíram esse efeito como aleatório apresentouse muito baixa, fornecendo estimativas praticamente nulas, 0,0025 e 0,0022. Apesar disso, a inclusão do efeito aleatório de linhagem citoplasmática no modelo estatístico de análise da produção de leite proporcionou melhor ajuste dos dados, explicando melhor a variação da produção de leite e fornecendo melhores estimativas dos parâmetros genéticos.
\end{abstract}

Palavras-chave: bovino de leite, herança citoplasmática, herdabilidade

\section{Effect of Cytoplasmic Lineage on Milk Yield of Caracu Cattle Breed}

\begin{abstract}
The objective of this research was to evaluate the effect of cytoplasmic lineage on milk yield of 11,163 records of Caracu cattle breed. The cytoplasmic lineage of each cow was traced back to the first female ancestor in the maternal line of its pedigree. The estimates of (co) variances and genetic parameters for milk yield including or not the components of maternal and cytoplasmic lineage effects, performed by univariate analyses were evaluated according to six different models using the derivative-free restricted maximum likelihood method. The cytoplasmic lineage variance estimate in the two models that included this random effect was very low, 0.0025 and 0.0022 . Therefore, the inclusion of the random cytoplasmic lineage effect in the milk yield statistical model better explained the milk yield variation and also produced better estimates of genetic parameters for this trait.
\end{abstract}

Key Words: cytoplasmic inheritance, dairy cattle, heritability

\section{Introdução}

Alguns autores (WAGNER, 1972; ROBISON, 1981) têm sugerido a existência de variabilidade genética materna devida ao DNA extranuclear, uma vez que os elementos citoplasmáticos, principalmente a mitocôndria, que contém DNA, são herdados somente da mãe pelo citoplasma do óvulo. A mitocôndria é transmitida intacta de geração a geração, representando, portanto, um potencial veículo de efeitos de linhagem citoplasmática. Dessa forma, a linhagem citoplasmática pode ser obtida traçando-se o pedigree dos animais, por meio da linha materna, até as fêmeas fundadoras do rebanho.

As mitocôndrias fornecem a maior parte da energia utilizada pelas células da glândula mamária e as diferenças qualitativas e quantitativas do mtDNA podem explicar parte das variações na produção de leite (BELL et al., 1985). Pelo fato de as mitocôndrias terem sua própria forma de herança, os métodos tradicionais de seleção não têm sido capazes de escolher os melhores genótipos mitocondriais. De forma semelhante, as avaliações genéticas que ignoram os efeitos citoplasmáticos podem produzir estimativas distorcidas dos valores genéticos dos animais, embora o efeito prático dessas distorções possa ser considerado muito pequeno (O'NEILL e VAN VLECK, 1989).

Um dos primeiros estudos a confirmar a herança citoplasmática como fonte significativa de variação na produção animal foi o de BELL et al. (1985). Trabalhando com dados da primeira lactação de 4461

\footnotetext{
1 Parte da Dissertação de Mestrado no programa de Pós-Graduação em Zootecnia, FCAV- UNESP, do primeiro autor, financiada pela FAPESP

2 Departamento de Zootecnia FCAV - UNESP - 14.884-900 - Jaboticabal - SP.

3 Aluna de doutorado do Programa de Pós-Graduação em Zootecnia - Produção Animal - FCAV-UNESP.

4 Bolsista da FAPESP. E.mail: lucripe@fcav.unesp.br

5 Bolsista do CNP. E.mail: saquei@fcav.unesp.br
} 
vacas da raça Holandesa e formando linhagens pela identificação das matriarcas do rebanho por pesquisa de pedigree, esses pesquisadores afirmaram que os efeitos de linhagem citoplasmática foram responsáveis por $2,0 \%$ da variância fenotípica para a característica produção total do leite e 3,5\% para porcentagem de gordura do leite. Resultados concordantes foram obtidos por TESS et al. (1987), que relataram efeitos citoplasmáticos significativos sobre a produção de leite de vacas Hereford. Entretanto, TESS e ROBISON (1990), reexaminando o trabalho e usando metodologia BLUP (Best Linear Unbisead Predictor), concluíram que efeitos aditivos atribuídos a touro e avô materno incluídos no modelo estavam confundidos com os de linhagem citoplasmática. Mais recentemente, ALBUQUERQUE et al. (1998), estudando os efeitos citoplasmáticos e aditivos direto e materno, verificaram para as características produção de leite e porcentagem de gordura do leite que a variância genética aditiva materna representou de 0,8 a $1,0 \%$; a covariância entre os efeitos genético direto e materno, de 0,7 a $2,5 \%$; e para os efeitos citoplasmáticos, valores de 3,4 e $2,3 \%$, respectivamente, da variância fenotípica.

Considerando-se a espécie bovina com suas diferentes raças e aptidões econômicas, as conclusões variam quanto à importância dos efeitos citoplasmáticos. Possivelmente, parte das diferenças obtidas nas pesquisas realizadas seja atribuída, principalmente, às diferenças de procedimentos estatísticos.

O objetivo do presente trabalho foi avaliar o efeito da linhagem citoplasmática sobre a produção total de leite das vacas de um rebanho Caracu.

\section{Material e Métodos}

Os dados para o presente estudo foram obtidos do arquivo zootécnico da fazenda Chiqueirão, situada no município de Poços de Caldas, MG, que se dedica à criação de bovinos da raça Caracu.

O rebanho Caracu da fazenda Chiqueirão iniciou-se em 1893, com a aquisição das primeiras matrizes e touros. Os animais foram adquiridos até a década de 40 , quando o rebanho foi fechado e passou a produzir seus próprios reprodutores.

A propriedade tem 1800 hectares, aproximadamente, de pastagens que podem ser divididas em duas partes distintas: campo e cultura. O primeiro constitui cerca de $30 \%$ das pastagens, predominando gramíneas nativas da região, enquanto nas áreas de cultura predominam pastagens de gordura (Melinis minutiflora, Pal. de Beauv), elefante (Pennisetum purpureum, Schum) e guiné (Pannicum maximum, Jacq.), além de áreas de cultura de inverno, azevém (Lolium perene).

A fazenda Chiqueirão possui duas maternidades e dez retiros onde os animais são ordenhados. A ordenha é feita manualmente, duas vezes ao dia, com o bezerro ao pé, e os bezerros ficam separados das mães no intervalo das ordenhas.

O leite proveniente de uma teta é deixado para o bezerro em cada ordenha. Os retiros acomodam de 36 a 75 vacas e o período de lactação é de, aproximadamente, 300 dias, ocasião em que é feita a desmama dos bezerros.

Para a análise dos dados, foram utilizadas 11.163 lactações de vacas nascidas no período de 1970 a 1995. A formação e editoração dos arquivos foram feitas no programa computacional SAS (1995).

Os anos de parto considerados variaram de 1973 a 1997 e as estações do ano foram divididas em quatro: primavera (setembro, outubro e novembro), verão (dezembro, janeiro e fevereiro), outono (março, abril e maio) e inverno (junho, julho e agosto), pois, na região onde localiza-se a fazenda, ocorrem diferenças marcantes entre estes períodos.

A variável grupo contemporâneo (GC) foi criada considerando-se ano e estação do parto. Foram eliminados GC com menos do que cinco indivíduos.

Um programa computacional, em linguagem FORTRAN, foi desenvolvido para a obtenção das linhagens citoplasmáticas. A linhagem citoplasmática foi obtida traçando-se o pedigree dos animais, pela linha materna, até as fêmeas fundadoras do rebanho, e foi estudada considerando-se o efeito genéticocitoplasmático como aleatório. O GC e a covariável duração da lactação (DL) foram incluídos em todos os modelos como efeitos fixos.

A variável produção de leite (PL) foi previamente corrigida para a idade da vaca ao parto, usando-se fatores de correção calculados para este efeito por CARVALHO DIAS (1997).

O efeito materno foi considerado, principalmente, pelo fato de a raça Caracu ser considerada de dupla aptidão e dos bezerros serem manejados ao pé da vaca durante toda a lactação.

Com o intuito de apresentar estimativas de (co)variâncias e parâmetros genéticos para a característica PL, incluindo-se ou não os componentes de efeito materno, de linhagem citoplasmática e de ambiente permanente, foram realizadas análises univariadas sob seis diferentes modelos, a saber: 
10 modelo: incluiu os efeitos fixos de GC e DL e os aleatórios genético aditivo direto, genético materno, de linhagem citoplasmática e de ambiente permanente;

2 o modelo: incluiu os efeitos fixos de GC e DL e os aleatórios genético aditivo direto, genético materno e de ambiente permanente;

3 o modelo: incluiu os efeitos fixos de GC e DL e os aleatórios genético aditivo direto, de linhagem citoplasmática e de ambiente permanente;

$4^{\mathrm{O}}$ modelo: incluiu os efeitos fixos de GC e DL e os aleatórios genético aditivo direto e de ambiente permanente;

5o modelo: incluiu os efeitos fixos de GC e DL e genético aditivo direto como aleatório;

6 - modelo: incluiu os efeitos fixos de GC e DL e como aleatórios os genético aditivo direto e genético materno.

Em termos matriciais, o modelo 1, o mais completo, pode ser descrito por:

$$
\mathbf{Y}=\mathbf{X} \beta+\mathbf{Z a}+\mathbf{M m}+\mathrm{Dlc}+\mathbf{W p e}+\mathbf{e}
$$

em que $\mathbf{Y}$ é o vetor de observações; $\boldsymbol{\beta}$, o vetor de efeitos fixos; a, o vetor de efeito genético aditivo direto dos animais; $\mathbf{m}$, o vetor de efeito genético aditivo materno; lc, o vetor de efeitos citoplasmáticos; pe, o vetor de efeito ambiental permanente; $\mathbf{e}$, o vetor de efeitos residuais, com $\mathbf{X}, \mathbf{Z}, \mathbf{M}, \mathbf{D}$ e $\mathbf{W}$ sendo as matrizes de incidência associadas aos efeitos apropriados a $\mathbf{Y}$. Para este modelo $\mathrm{E}[\mathrm{Y}]=\mathrm{X} \beta, \mathrm{E}[\mathrm{a}]=0$, $\mathrm{E}[\mathrm{m}]=0, \mathrm{E}[\mathrm{lc}]=0$ e $\mathrm{E}[\mathrm{e}]=0 ; \operatorname{Var}[\mathrm{a}]=\mathrm{A} \sigma^{2}{ }_{\mathrm{a}}$, $\operatorname{Var}[\mathrm{m}]=\mathrm{A} \sigma^{2}{ }_{\mathrm{m}}, \operatorname{Cov}[\mathrm{a}, \mathrm{m}]=\mathrm{A} \sigma_{\mathrm{am}}, \operatorname{Var}[\mathrm{lc}]=\mathrm{I}_{\mathrm{NC}} \sigma^{2}{ }_{1 \mathrm{c}}$, $\operatorname{Var}[\mathrm{pe}]=\mathrm{I}_{\mathrm{NP}} \sigma_{\mathrm{pe}}^{2}$, e $\operatorname{Var}[\mathrm{e}]=\mathrm{I}_{\mathrm{N}} \sigma_{\mathrm{e}}^{2}$, NC é o número de linhas maternas; NP, o número de vacas; N, o número de registros; $\mathrm{A}$, o numerador da matriz de parentesco; I, uma matriz identidade e $\sigma_{a}^{2}, \sigma^{2}{ }_{m}, \sigma^{2}{ }_{l c}$, $\sigma_{\text {pe }}^{2}, \sigma_{\mathrm{e}}^{2}$ e $\sigma_{\mathrm{am}}$, os componentes de variância aditivo direto, aditivo materno, de linhagem citoplasmática, de ambiente permanente, residual e o componente de covariância entre os efeitos aditivo direto e materno, respectivamente. Assume-se que os vetores a e m não são correlacionados com os vetores lc, pe e e.

As estimativas dos componentes de (co)variâncias e dos parâmetros genéticos foram obtidos por máxima verossimilhança restrita livre de derivada (DFRML "derivative-free restricted maximum likelihood"), utilizando-se o programa computacional MTDFREML ("multiple trait derivative-free restricted maximum likelihood"), descrito por BOLDMAN et al. (1993). O critério de convergência considerado foi de $10^{-9}$.

A herdabilidade do efeito genético total $\left(\mathrm{h}^{2} \mathrm{~T}\right)$, isto é, a regressão do genótipo total do animal (direto + materno) sobre o fenótipo, foi calculada como proposto por WILLHAM (1972).
Para verificar o efeito da inclusão da linhagem citoplasmática no modelo de análise, realizou-se o teste de razão de verossimilhança, utilizando-se os modelos 1 (com o efeito de linhagem citoplasmática) e 2 (sem o efeito de linhagem citoplasmática).

Neste teste, segundo FREUND e WALPOLE (1980), define-se $\lambda$ como a razão de verossimilhança calculada como segue:

$$
\lambda=\mathrm{Lc} / \mathrm{Lr}
$$

em que Lc é valor de máxima verossimilhança do modelo completo (modelo 1, com o efeito de linhagem citoplasmática) e Lr, valor de máxima verossimilhança do modelo reduzido (modelo 2 , sem o efeito da linhagem citoplasmática).

Ainda, segundo esses autores, para valores grandes de $n$, a distribuição de $-2 \log \lambda$ aproxima-se, em condições gerais, a uma distribuição de chi-quadrado $\left(\chi^{2}\right)$ com 1 grau de liberdade, i.e.:

$$
-2 \log \lambda \sim \chi^{2} \alpha, v
$$

em que $\alpha$ é nível de significância e $v$, grau de liberdade (1).

O cálculo de - $2 \log \lambda$ pode ser simplificado aplicando-se a diferença entre -2logLc e - $2 \log \mathrm{Lr}$, uma vez que:

$$
\begin{aligned}
& \lambda=\mathrm{Lc} / \mathrm{Lr}=\mathrm{Lc}_{\mathrm{L}} \mathrm{Lr}^{-1} \\
& \Rightarrow \log \lambda=\log \mathrm{Lc}-\log \mathrm{Lr} \\
& \Rightarrow-2 \log \lambda=(-2 \log \mathrm{Lc})-(-2 \log \mathrm{Lr})
\end{aligned}
$$

Assim, quando $-2 \log \lambda \geq \chi_{\alpha, v}^{2}$, pode-se afirmar que o efeito é significativo.

\section{Resultados e Discussão}

A média observada para produção de leite foi de $2033,57 \mathrm{~kg} /$ lactação, variando de 167,97 a $4358,00 \mathrm{~kg} / \mathrm{lactação.} \mathrm{Esta} \mathrm{média} \mathrm{aproxima-se} \mathrm{à} \mathrm{obtida,}$ no mesmo rebanho, por FRAGA (1992), que foi 1770,54 kg/lactação.

As estimativas dos componentes de (co)variância e dos parâmetros genéticos para a produção de leite são apresentadas na Tabela 1 e as estimativas dos componentes de variância, como porcentagem da variância fenotípica total, na Tabela 2 .

Observa-se que, quando o efeito aleatório de linhagem citoplasmática foi incluído (modelo 1), a estimativa de variância genética aditiva $\left(\sigma_{\mathrm{a}}^{2}\right)$ diminuiu em relação aos demais modelos e a variância de ambiente permanente $\left(\sigma_{\mathrm{pe}}{ }^{2}\right)$ foi responsável por cerca de $30,94 \%$ da variação total da produção de leite. Pelo exposto nas Tabelas 1 e 2, pode-se inferir que parte da $\sigma_{\mathrm{a}}^{2}$ obtida sob o modelo $2\left(39610,4471 \mathrm{~kg}^{2}\right)$ foi explicada pelos efeitos de linhagem citoplasmática e de ambiente permanente da mãe (modelo 1). Veri- 
fica-se, também, que a variância de linhagem citoplasmática $\left(\sigma_{1 \mathrm{c}}{ }^{2}\right)$, apesar do baixo percentual em relação à variância fenotípica total $\left(\sigma_{\mathrm{p}}^{2}\right)$, afetou $\sigma_{\mathrm{pe}}{ }^{2}$, inflacionando esta estimativa.

A análise sob o modelo mais completo (modelo 1) forneceu valor da estimativa de $\sigma_{\mathrm{a}}^{2}$ menor que as obtidas sob os demais modelos. Ignorando o efeito genético materno (modelo 3 ), houve pequeno aumento da $\sigma_{\mathrm{pe}}^{2}$ e da variância residual $\left(\sigma_{\mathrm{e}}^{2}\right)$ em relação ao modelo 2. Os modelos 1 e 3 foram muito semelhantes, indicando, portanto, pouca influência doefeito materno, de modo geral.

A covariância entre os efeitos aditivo direto e materno $\left(\sigma_{\mathrm{am}}\right)$ foi muito baixa em todos os modelos, fornecendo estimativas, praticamente, nulas de correlação entre os efeitos aditivo direto e materno $\left(\mathrm{r}_{\mathrm{am}}\right)$

No modelo 4, que incluiu apenas os efeitos genético aditivo direto e de ambiente permanente, é possível verificar maior valor de $\sigma^{2}$ a em relação aos modelos 1, 2 e 3, como era de se esperar, já que a $\sigma^{2}{ }_{a}$ sob esse modelo (modelo 4) continha, além da variação devida aos efeitos genéticos direto e materno, a variação causada pelo componente de $\sigma^{2}{ }_{\text {lc }}$. Verifica-se que a $\sigma_{\text {pe }}^{2}$ se apresentou menor que nos modelos 1 e 3 e semelhante ao modelo 2 .

Comparando-se os modelos 2 e 4 verifica-se que $\sigma_{a}^{2}, \sigma_{\text {pe }}^{2}$ e $\sigma_{\text {e }}^{2}$ foram muitos semelhantes, demonstrando a pouca influência do efeito materno.

As estimativas de $\sigma_{a}^{2}$ e herdabilidade do efeito direto $\left(\mathrm{h}_{\mathrm{a}}{ }^{2}\right)$ obtidas sob os modelos 5 e 6 foram, substancialmente, mais altas, quando comparadas com as obtidas sob os outros modelos, demonstrando a grande importância do ambiente permanente para a característica em questão.

Quando somente os efeitos genético aditivo direto e de ambiente permanente da mãe foram incluídos (modelo 4), a estimativa de $\sigma^{2}$ diminuiu em relação aos modelos 5 e 6 (sem o efeito de ambiente permanente) e a $\sigma^{2}$ pe foi responsável por $29,55 \%$ da variação total da produção de leite. Na verdade, parte da $\sigma^{2}$ obtida sob os modelos 5 e $6\left(129303,9273 \mathrm{~kg}^{2} \mathrm{e}\right.$ $129312,9517 \mathrm{~kg}^{2}$ ) foi explicada pelo efeito de ambiente permanente da mãe (modelo 4). A inclusão deste reduziu a estimativa de $\mathrm{h}_{\mathrm{a}}{ }^{2} \mathrm{e}$, conseqüentemente, de herdabilidade total $\left(\mathrm{h}_{\mathrm{T}}{ }^{2}\right)$, em 63,6\% em relação aos modelos 5 e 6 .

Analisando o modelo 6, que considerou o efeito

Tabela 1 - Estimativas dos componentes de (co)variância $\left(\mathrm{em} \mathrm{kg}^{2}\right)$ e parâmetros genéticos para a produção de leite de vacas da raça Caracu

Table 1 - Estimates of (co)variances components (in $\mathrm{kg}^{2}$ ) and genetic parameters for milk yield of Caracu cows

\begin{tabular}{lcccccc}
\hline Componentes & \multicolumn{7}{c}{ Modelos } \\
& \multicolumn{7}{c}{ Models } \\
\cline { 2 - 7 } & 1 & 2 & 3 & 4 & 5 & 6 \\
\hline$\sigma^{2}{ }_{\mathrm{a}}$ & 36356,4412 & 39610,4471 & 36507,0479 & 39615,7468 & 129303,9273 & 129312,9517 \\
$\sigma^{2}{ }_{\mathrm{m}}$ & 202,1808 & 0,1868 & - & - & - & 0,4855 \\
$\sigma^{2}{ }_{\mathrm{am}}$ & 2,1033 & 3,5595 & - & - & - & $-0,7875$ \\
$\sigma^{2}{ }_{\mathrm{lc}}$ & 0,0025 & - & 0,0022 & - & - & - \\
$\sigma^{2}{ }_{\mathrm{pe}}$ & 58561,9825 & 56085,7727 & 58782,2141 & 56078,3109 & - & - \\
$\sigma^{2}{ }_{\mathrm{e}}$ & 94098,1035 & 94042,4853 & 94092,9797 & 94041,7824 & 96090,4090 & 96107,8954 \\
$\sigma^{2}{ }_{\mathrm{p}}$ & 189220,8138 & 189742,4514 & 189382,2439 & 189735,8401 & 225394,3363 & 225420,5451 \\
$\mathrm{~h}^{2}{ }_{\mathrm{a}}$ & 0,1921 & 0,2087 & 0,1927 & 0,2088 & 0,5737 & 0,5736 \\
$\mathrm{~h}^{2}{ }_{\mathrm{m}}$ & 0 & 0 & - & - & - & 0 \\
$\mathrm{~h}^{2}{ }_{\mathrm{T}}$ & 0,1932 & 0,2088 & 0,1927 & 0,2088 & 0,5737 & 0,5737 \\
$\mathrm{r}_{\mathrm{am}}$ & 0 & 0,04 & - & - & - & 0 \\
\hline
\end{tabular}

$-2 \log \lambda=2,288649 *$

$\overline{\sigma^{2}}, \sigma^{2}{ }_{a}, \sigma_{m}^{2}, \sigma_{l c}^{2} \sigma_{p e}^{2}, \sigma_{p}^{2}, \sigma_{a m}, h^{2}{ }_{a}, h^{2}{ }_{m}, h^{2}{ }_{T}, r_{a m}$ e -2log $\lambda=$ componentes de variância residual, aditivo direto, aditivo materno, de linhagem citoplasmática, de ambiente permanente, fenotípica total, componente de covariância entre os efeitos aditivos direto e materno, herdabilidade do efeito direto, herdabilidade do efeito materno, herdabilidade total e correlação genética entre os efeitos direto e materno -2 log da razão de verossimilhança, respectivamente. ${ }^{*}=$ significativo $(\mathrm{P}<0,13)$.

$\sigma^{2}{ }_{e}, \sigma^{2}{ }_{a}, \sigma^{2}{ }_{m}, \sigma^{2}{ }_{l c} \sigma^{2}{ }_{p e}, \sigma^{2}, \sigma_{a m}, h^{2}{ }_{a}, h^{2}{ }_{m}, h^{2}{ }_{T}, r_{\text {am }}$ e-2log $\lambda=$ residual variance, direct additive genetic variance, maternal additive genetic variance, cytoplasmic lineage variance, maternal permanent environmental variance, phenotypic variance, covariance between direct and maternal effects, direct heritability, maternal heritability, total heritability and genetic correlation between direct and maternal effects and -2 log likelihood ratio, respectively. ${ }^{*}=$ significant $(P<0,13)$. 
Rev. bras. zootec.

Tabela 2 - Estimativas dos componentes de variância, para a produção de leite de vacas da raça Caracu, expressas como porcentagem da variância fenotípica total

Table 2 - Estimates of variances components for milk yield of Caracu cows expressed as a percentage of the total phenotipe variance

\begin{tabular}{|c|c|c|c|c|c|c|}
\hline \multirow[t]{2}{*}{$\begin{array}{l}\text { Componentes } \\
\text { Components }\end{array}$} & \multicolumn{5}{|c|}{$\begin{array}{l}\text { Modelos } \\
\text { Models }\end{array}$} & \multirow[b]{2}{*}{6} \\
\hline & 1 & 2 & 3 & 4 & 5 & \\
\hline$\sigma_{\mathrm{a}}^{2}(\%)$ & 19,21 & 20,87 & 19,27 & 20,88 & 57,37 & 57,36 \\
\hline$\sigma_{\mathrm{m}}^{2}(\%)$ & 0,10 & $9,84 \times 10-5$ & - & - & - & $2,15 \times 10-4$ \\
\hline$\sigma^{2}{ }_{1 \mathrm{c}}(\%)$ & $1,32 \times 10-6$ & - & $1,16 \times 10-6$ & - & - & - \\
\hline$\sigma_{\text {pe }}^{2}(\%)$ & 30,94 & 29,55 & 31,04 & 29,55 & - & - \\
\hline$\sigma_{\mathrm{e}}^{2}(\%)$ & 49,73 & 49,56 & 49,68 & 49,56 & 42,63 & 42,63 \\
\hline$\sigma_{p}^{2}(\%)$ & 100,00 & 100,00 & 100,00 & 100,00 & 100,00 & 100,00 \\
\hline
\end{tabular}

$\sigma^{2}{ }_{e}, \sigma_{a}^{2}, \sigma_{m}^{2}, \sigma_{\text {lc }}^{2} \sigma_{p e}^{2}$ e $\sigma_{p}^{2}=$ componentes de variância residual, aditivo direto, aditivo materno, de linhagem citoplasmática, de ambiente permanente e fenotípica total, respectivamente.

$\sigma^{2}{ }_{e}, \sigma^{2}{ }_{a}, \sigma^{2}{ }_{m}, \sigma_{l c}^{2} \sigma_{p e}^{2}, \sigma^{2}{ }_{p}=$ residual variance, direct additive genetic variance, maternal additive genetic variance, cytoplasmic lineage variance, maternal permanent environmental variance and phenotypic variance, respectively.

genético materno e excluiu o de ambiente permanente, a estimativa de $\sigma_{\mathrm{a}}^{2}$ foi semelhante à obtida no modelo 5 , que incluiu somente o efeito aditivo direto, demonstrando a pouca importância do efeito materno para a característica estudada.

Apesar de o modelo 2 não incluir o efeito de linhagem citoplasmática, a variação devida a este efeito ficou, na sua maioria, contida na $\sigma_{\mathrm{a}}{ }^{2}$, explicando o fato de a estimativa de $\mathrm{h}_{\mathrm{T}}{ }^{2}$ obtida sob este modelo ser semelhante às verificadas sob os modelos $1 \mathrm{e} 3$, que incluíram o efeito de linhagem citoplasmática.

A $\sigma_{1 \mathrm{c}}{ }^{2}$ nos dois modelos que incluíram o efeito aleatório de linhagem citoplasmática apresentou-se muito baixa, fornecendo estimativas praticamente nulas $(0,0025$ e 0,0022 para os modelos 1 e 3, respectivamemte). Entretanto, a inclusão do efeito de linhagem citoplasmática no modelo de análise parece ter maior impacto sobre a $\sigma_{\mathrm{pe}}{ }^{2}$, que, por sua vez, na ausência de $\sigma_{\mathrm{lc}}{ }^{2}$, inflaciona a $\sigma_{\mathrm{a}}{ }^{2}$. Possivelmente, isso se deve ao fato de o efeito de linhagem citoplasmática ser parte da variação devida ao ambiente permanente. Essas estimativas de $\sigma_{\text {lc }}^{2}$ aproximam-se da encontrada por RORATO et al. (1999), que, trabalhando com 138869 lactações de 68063 vacas Holandesas, nos Estados Unidos, verificaram estimativa de componente de variância para o efeito genético citoplasmático igual a 0,011 .

Verifica-se, na Tabela 1, que o valor de $-2 \log \lambda$ calculado para a característica PL se mostrou significativo a $13 \%$ de probabilidade. Assim, os resultados encontrados, neste estudo, indicam pequena, mas considerável, contribuição do efeito de linhagem citoplasmática para a característica produção de leite das vacas do rebanho Caracu estudado.

Os resultados obtidos evidenciaram a pequena influência do efeito genético materno e a expressiva contribuição do efeito de ambiente permanente em bovinos leiteiros, como já era esperado. Embora a $\sigma^{2}{ }_{l c}$ encontrada nos modelos que incluíram a linhagem citoplasmática como efeito aleatório apresentasse estimativas muito pequenas, a inclusão deste efeito nos modelos de análise estatística poderia ser justificada por proporcionar melhor ajuste dos dados, explicando melhor a variação da produção de leite e propiciando estimativas mais fiéis dos parâmetros genéticos.

\section{Conclusões}

A inclusão do efeito aleatório de linhagem citoplasmática no modelo de PL proporcionou melhor ajuste dos dados, explicando melhor a variação da produção de leite e fornecendo melhores estimativas dos parâmetros genéticos.

A não-inclusão do efeito de ambiente permanente no modelo estatístico acarretou valores superestimados de $\sigma_{\mathrm{a}}^{2}$.

$\mathrm{O}$ efeito materno pode ser desconsiderado na análise da PL, mesmo para raças de dupla aptidão. 


\section{Agradecimento}

Ao zootecnista M.S. André Carvalho Dias (Fazenda Chiqueirão), pela cessão dos dados, e à FAPESP, pelo suporte financeiro.

\section{Referências Bibliográficas}

ALBUQUERQUE, L.G., KEOWN, J.F., VAN VLECK,L.D. 1998. Variances of direct and maternal genetic and cytoplasmic effects for yields and fat percentage. J. Dairy Sci., 81:544-49.

BELL, B.R., McDANIEL, B.T., ROBISON, O.W. 1985. Effects of cytoplasmic inheritance on production traits of dairy cattle. J. Dairy Sci., 68:2038.

BOLDMAN, K.G., KRIESE, L.A., VAN VLECK, L.D. et al. 1993. A Manual for Use of MTDFREML. USDA-ARS. Clay Center, NE. 120p.

CARVALHO DIAS, A.S. Estudo de características produtivas e de permanência em um rebanho de bovinos da raça Caracu. Jaboticabal, SP: FCAV, 1997. 65p. Dissertação (Mestrado em Zootecnia) - Faculdade de Ciências Agrárias e Veterinárias, Universidade Estadual Paulista, 1997.

FRAGA, A.B. Aspectos genéticos e fenotípicos da produção de leite em um rebanho de bovinos da raça Caracu. Jaboticabal, SP: FCAV, 1992. 34p. Monografia (Graduação em Zootecnia) Faculdade de Ciências Agrárias e Veterinárias, Universidade Estadual Paulista, 1992.

FREUND, J.F., WALPOLE, R.E. 1980. Mathematical statistics, 3.ed. New Jersey: Prentice-Hall. 548p.

O'NEILL, K., VAN VLECK, D. L. 1989. Potencial of cytoplasmic effects for selection in dairy cattle. J. Dairy Sci., 71:3390-3398.
ROBISON, O.W. 1981. The influence of maternal effects on the efficiency of selection, A review. Livest. Prod. Sci., 8(2):121-137.

RORATO, P.R.N., KEOWN, J.F., VAN VLECK, L.D. 1999. Variance caused by cytoplasmic line and sire by herd interaction effects for milk yield considering estimation bias. J. Dairy Sci., 82:1574-1580.

SAS, 1995. Institute Inc. SAS Language Guide, v. 6.03 Edition, Care, NC: SAS INSTITUTE INC. 530p.

TESS, M.W., ROBISON, O.W. 1990. Evaluation of cytoplasmic genetics effects in beef cattle using an animal model. J. Anim. Sci., 68(7):1899-1909.

TESS, M.W., REODECHA, C., ROBISON, O.W. 1987. Cytoplasmic genetics effects on preweaning growth and milk yield in Hereford cattle. J. Anim. Sci., 65(3):675-684.

WAGNER, R.P. 1972. The role of maternal effects in animal breeding: II. Mitochondria e animal inheritance. J. Anim. Sci., 35(6): 1280-1286.

WILLHAM, R.L. 1972. The role of maternal effects in animal breeding: III-Biometrical aspects of maternal effects in animals. J. Anim. Sci., 35:1288-1302.

Recebido em: 16/06/00

Aceito em: 08/09/00 